-Supporting Information for Publication-

\title{
High-Throughput Quantification of Surface Protein Internalization and Degradation
}

\author{
Jakob C. Stüber ${ }^{1 \#}$, Florian Kast ${ }^{1 \#}$, and Andreas Plückthun ${ }^{1 *}$ \\ ${ }^{1}$ Department of Biochemistry, University of Zurich, Winterthurerstrasse 190, CH-8057 Zurich, \\ Switzerland \\ *Corresponding author, e-mail: plueckthun@bioc.uzh.ch, fax: +41-44-63 55712. \\ \#J.C.S. and F.K. contributed equally to this work. \\ Contents \\ SI Methods \\ SI Figures \\ SI References
}




\section{SI Methods}

\section{Generation of T-REx cell lines with the Flp-In system}

Stable cell lines for EGFR, HER2, and EpCAM were generated using the FIp-In T-REx system (Thermo Fisher, cat. no. K650001). In brief, we used PCR to amplify the coding sequences from plasmids containing EGFR (Addgene, cat. no. 11011), HER2 (Mammalian Gene Collection, GenBank accession number BC156755.1), and EpCAM ${ }^{1}$. Next, we inserted the amplicons into a modified version of the pcDNA5/FRT/TO plasmid (contained in the Flp-In T-REx kit), which contains an expression cassette in which the HaloTag7 $(\mathrm{HT})$ is fused to the N-terminus of the protein of interest (POI), connected by a short glycine-serine linker, via standard cloning techniques.

Sub-confluent cultures of Flp-In T-REx 293 cells (Thermo Fisher, cat. no. R78007) were transfected with pcDNA5/FRT/TO-derived plasmids, encoding the HT-POI fusions, and the Flp recombinase vector pOG44 (included in the Flp-In TREx kit), using TransIT-293 (Mirus Bio, cat. no. 2700 ) according to the instructions by the manufacturers. Stable clones were selected by $150 \mathrm{mg} \mathrm{ml}^{-1}$ hygromycin B. Single clones were isolated, expanded, and analyzed for inducible expression of the HT-POI fusion to obtain stable HEK-TREx_HT-POI cell lines (Figure S-1).

\section{Detailed protocol for high-throughput treatment, labeling, and flow cytometry}

Note: For items considered critical supplier information is provided.

\section{General cell culture}

Culture HEK-TREx_HT-POI (protein of interest) cell lines in Dulbecco's Modified Eagle Medium (DMEM, Sigma, cat. no. D6429) supplemented with 10\% "tetracycline free" fetal calf serum (Bioconcept, cat. no. 2-01F28-I), 1\% (v/v) penicillin-streptomycin solution (Sigma-Aldrich, cat. no. P0781), and $150 \mu \mathrm{g} \mathrm{ml}^{-1}$ hygromycin B (Invitrogen, cat. no. 10687-10).

CAUTION: Hygromycin B is hazardous to health and corrosive. Perform all handling and disposal of biological materials according to the regulations of your institution.

Reagents and materials

- All treatment agents to be studied

- Dulbecco's phosphate buffered saline (DPBS)

- PBSA: DPBS supplemented with $0.1 \%(\mathrm{w} / \mathrm{v}) \mathrm{NaN}_{3}$ (can be stored for several weeks at 4 $\left.{ }^{\circ} \mathrm{C}\right)$

CAUTION: $\mathrm{NaN}_{3}$ is highly toxic, hazardous to health, dangerous for the environment, and capable of forming explosive hydrazoic acid in acidic solution. Handle solutions with special care and dispose waste according to regulations of your institution.

- PBSBA: PBSA supplemented with $1 \%(\mathrm{w} / \mathrm{v})$ bovine serum albumin (can be stored for several weeks at $4{ }^{\circ} \mathrm{C}$ )

- $4 \%(\mathrm{w} / \mathrm{v})$ paraformaldehyde (PFA) dissolved in DPBS (stored at $-20{ }^{\circ} \mathrm{C}$ ) 
CAUTION: Formaldehyde is hazardous to health, corrosive, and flammable. Handle with special care and dispose waste according to regulations of your institution.

- V-bottom propylene 96-well plates (cat. no. 651201, Greiner Bio-One)

- Deep well plates (Thermo Fisher, cat. no. AB-0661)

- Trypsin-EDTA solution

- Complete medium (see above)

- HTL-TMR (Promega, cat. no. G8252) and HTL-AF660 (Promega, cat. no. G8472)

- Fixable Aqua Dead Cell Stain Kit (Thermo Fisher, L34966). Alternatively, another dead cell stain may be chosen to be detected in another, non-interfering channel, depending on the available lasers and filter sets of the flow cytometry instrument at your disposal

- Tissue culture 96-well plate

- Doxycycline hyclate ${ }^{1}$ (Applichem, cat. no. A2951.0005) dissolved in ultrapure water to yield a $1 \mathrm{mg} \mathrm{ml}^{-1}$ stock solution (stored at $-20{ }^{\circ} \mathrm{C}$ )

- Multichannel pipette reagent reservoirs

- Microplate cover film

\section{Equipment}

- Tissue culture incubator with $\mathrm{CO}_{2}$ control

- Multichannel pipette (8 or 12 channels)

- Laboratory centrifuge with inserts suitable for 96-well plates

- Microplate shaker

- Bright-field microscope

- Flow cytometer with high-throughput sample loader (e.g., LSR II Fortessa (BD)) and lasers and filter sets to record the signals of HTL-TMR (e.g., ex. $561 \mathrm{~nm}$ and 586/15 nm filter), HTL-AF660 (e.g., ex. 640 nm and 670/14 nm filter), and Aqua fixable dead cell stain (e.g., ex. $405 \mathrm{~nm}$ and 525/50 nm filter) or another non-interfering dead cell stain

\section{Day 1: Cell seeding}

Harvest HEK-TREx_HT-POI cells by trypsinization. Seed $100 \mu \mathrm{l}$ per well of a $2 \cdot 10^{5} \mathrm{ml}^{-1}$ cell solution into 96-well tissue culture plate. When creating the layout of your plate include 3 control conditions not treated by any agent in duplicates or triplicates for rescaling into true relative abundances (see below): Non-labeled cells, single-labeled cells (HTL-TMR only), and doublelabeled cells. Allow at least $18 \mathrm{~h}$ for the cells to adhere fully to the plate before inducing expression of HT-POI.

\footnotetext{
${ }^{1}$ Hyclate is an abbreviation for hydrochloride hemiethanolate hemihydrate, which means that each molecule of doxycycline comes with $1 \cdot \mathrm{HCl}$ and $0.5 \cdot \mathrm{CH}_{3} \mathrm{CH}_{2} \mathrm{OH}$ and $0.5 \cdot \mathrm{H}_{2} \mathrm{O}$ in the crystal.
} 


\section{Day 2: Induction}

Induce HT-POI expression by addition of $50 \mu \mathrm{l}$ of a 3 -fold $(3 \mu \mathrm{g} \mathrm{ml}-1)$ doxycycline stock in complete medium to all wells. After induction, allow at least $12 \mathrm{~h}$ for surface POI level to reach a steady state.

\section{Day 3-4: Treatment}

Using a sterile (deep) multi-well plate, prepare a master plate containing 4-fold stocks of treatment agents for treatment, preferably diluted in DPBS or serum-free medium and cover the plate. Treat respective wells by addition of $50 \mu \mathrm{l}$ of the 4 -fold stock, adding DPBS or serumfree medium to the control wells. We consider 1-24 $\mathrm{h}$ to be suitable treatment times to be investigated by this assay.

Day 4: Labeling and fixation

1. Prior to completion of the desired treatment times, thaw HTL-TMR, HTL-AF660, and PFA at room temperature.

2. Equilibrate trypsin, complete medium, and DPBS at $37^{\circ} \mathrm{C}$.

3. Prepare a master plate (depending on your plate layout in deep well plates) containing a 9-fold stock solution of HTL-AF660 (3600 nM) and a 10-fold HTL-TMR stock (2500 nM) in DPBS in the respective positions and cover the plate with microplate cover film or laboratory tape.

4. After completion of the desired treatment times, add $25 \mu \mathrm{l}$ of the 9-fold HTL-AF660 solution to the respective wells for a final concentration of $400 \mathrm{nM}$ and incubate for $15 \mathrm{~min}$ at $37^{\circ} \mathrm{C}$.

5. Thaw the fixable dead cell stain and DMSO at room temperature.

6. Add $25 \mu \mathrm{l}$ of the 10 -fold HTL-TMR solution to the respective wells for a final concentration of $250 \mathrm{nM}$ and a total volume of $250 \mu \mathrm{l}$, and incubate for $15 \mathrm{~min}$ at $37^{\circ} \mathrm{C}$.

7. Prepare fixable dead cell staining solution in DPBS in a master plate, according to the instructions of the manufacturer.

8. Centrifuge plate(s) for $1 \mathrm{~min}$ at $800 \times \mathrm{g}$, remove the buffer by inverting the plate swiftly above a suitable liquid waste container, and add $250 \mu$ LPBS. Repeat once, for a total of two wash cycles.

9. Centrifuge plate(s) for $1 \mathrm{~min}$ at $800 \times \mathrm{g}$, remove the buffer by inverting the plate swiftly above a suitable liquid waste container, and add $150 \mu$ trypsin-EDTA per well. Incubate at $37^{\circ} \mathrm{C}$ and occasionally gently rock the plate until complete cell detachment can be observed (typically, 10-15 min) in the bright field microscope.

10. Add $100 \mu \mathrm{l}$ of complete medium per well to quench trypsin in all wells.

11. Using fresh tips per row (or column, respectively), re-suspend the cells by pipetting up and down 10 times and transfer the cell suspension to $\mathrm{V}$-bottom plates.

12. Centrifuge plate(s) for $1 \mathrm{~min}$ at $800 \times \mathrm{g}$, remove the buffer by inverting the plate swiftly above a suitable liquid waste container, and add $200 \mu$ LPBS per well. 
13. Centrifuge plate(s) for $1 \mathrm{~min}$ at $800 \times \mathrm{g}$, remove the buffer by inverting the plate swiftly above a suitable liquid waste container, and add $100 \mu \mathrm{l}$ per well of the fixable dead cell stain. Incubate for $30 \mathrm{~min}$ at room temperature.

14. Centrifuge plate(s) for $1 \mathrm{~min}$ at $800 \times \mathrm{g}$, remove the buffer by inverting the plate swiftly above a suitable liquid waste container, and add $200 \mu$ DPBS per well.

15. Centrifuge plate(s) for $1 \mathrm{~min}$ at $800 \times \mathrm{g}$, remove the buffer by inverting the plate swiftly above a suitable liquid waste container, and add $200 \mu \mathrm{l}$ 4\% PFA per well. Re-suspend the cells by shaking for 10-30 s on a microplate shaker at $\sim 800 \mathrm{~min}^{-1}$ and incubate for $20 \mathrm{~min}$ at room temperature.

16. Centrifuge plate(s) for $1 \mathrm{~min}$ at $800 \times \mathrm{g}$, remove the buffer by inverting the plate swiftly above a suitable liquid waste container, and add $200 \mu$ I PBSBA per well.

17. Centrifuge plate(s) for $1 \mathrm{~min}$ at $800 \times \mathrm{g}$, remove the buffer by inverting the plate swiftly above a suitable liquid waste container, and add $200 \mu$ I PBSA per well. Re-suspend the cells by shaking for $10-30 \mathrm{~s}$ on a microplate shaker at $\sim 800 \mathrm{~min}^{-1}$. Cover the plate with microplate sealing film. The plates can be stored for several days at $4{ }^{\circ} \mathrm{C}$ until analyzed by flow cytometry.

\section{Day 4 or later: Flow cytometry}

Measure the samples according to the operating procedures for use of the flow cytometer including the high-throughput sampler provided by your institution. Depending on the cell concentration and your instrument, measurement parameters need to be adjusted. We recommend five mixing cycles with a mixing volume of $100 \mu \mathrm{l}$, a mixing speed of $250 \mu \mathrm{s} \mathrm{s}^{-1}$, and a measurement flow of $3 \mu \mathrm{s} \mathrm{s}^{-1}$ as starting values. Using the control samples and small (e.g., $10 \mu \mathrm{l}$ ) sample volumes, adjust the instrument such that a significantly increased TMR signal (due to strong internalization) is well within the dynamic range. In a dead cell-stain-vs.-forward scatter (area) plot, draw a gate around the dead cell-stain negative cell population, excluding debris at lower forward scatter values. Furthermore, gate the obtained population for singlets in a forward scatter area-vs.-forward scatter height plot. We typically record $10^{3}$ (minimum) to $10^{4}$ cells in the singlet gate per well from $50 \mu$ sample volume or less.

\section{Data scaling}

Since the detection efficiency of the flow cytometry instrument in the channels for AF660 and TMR is different, the data have to be scaled in order to obtain relative abundances. Only then surface protein (detected in the AF660 channel) and internal protein (detected in the TMR channel) can be properly compared. To this end, a control sample (utr.,s.) is used in which the first (HTL-AF660-labeling) step is omitted. Thus, all (surface and internal) HaloTag molecules will react with HTL-TMR in this "single" (s.) labeling procedure (Figure 1(a)). Mean fluorescence intensities (MFI) of typically 1000-10,000 cells were calculated. For the TMR channel, 
the normalized (feature-scaled) signal $S_{\text {TMR }}$ of any sample is obtained by relating it to a singlelabeled untreated control sample (utr.,s.), with the background subtracted from both of them:

$$
S_{\mathrm{TMR}}(\text { sample })=\frac{M F I_{\mathrm{TMR}}(\text { sample })-M F I_{\mathrm{TMR}} \text { (utr.,unlab.) }}{\left.M F I_{\mathrm{TMR}} \text { (utr., s. }\right)-M F I_{\mathrm{TMR}} \text { (utr.,unlab.) }}
$$

where utr.,unlab. represents an untreated, unlabeled control (which shows only autofluorescence).

For the double labeling ("d.", Figure 1(a)), the first, surface-labeling step with cell-impermeable dye is virtually complete, i.e., almost all reactive HaloTag enzymes at the surface are conjugated to a HTL-AF660 molecule after 15-20 min (Figure 1(b), compare raw signals of surface stain only with the double stain). We can thus define the normalized surface signal $S_{A F 660}$ in an analogous way:

$$
S_{\mathrm{AF} 660}(\text { sample })=\frac{M F I_{\mathrm{AF} 660}(\text { sample })-M F I_{\mathrm{AF} 660}(\text { utr.,unlab. })}{M F I_{\mathrm{AF} 660}(\text { utr., d. })-M F I_{\mathrm{AF} 660}(\text { utr.,unlab. })}
$$

Since internal receptor cannot be stained by HTL-AF660, even if only this dye was used in a single-stain procedure, the $S_{\mathrm{AF} 600}$ signal of any sample can be related to the surface signal from a double-labeled control (utr., $d$.): the difference from the single to the double labeling procedure in the TMR channel, $\Delta S_{\text {TMR, }}$ corresponds exactly to the number of molecules blocked by the first, surface-specific step. This number of molecules is also reflected by the signal in the AF660 channel in the same double labeling experiment:

$$
\Delta S_{\mathrm{TMR}}=S_{\mathrm{TMR}}(\text { utr.,s. })-S_{\mathrm{TMR}}(\text { utr., d. })=S_{\mathrm{AF660,scaled}}(\text { utr.,d. })
$$

A correction factor $C_{A}$ relates the measured intensity $S_{A F 660}$ (utr.,d.) (which is recorded in the AF660 channel) to $S_{A F 660, \text { scaled }}($ utr.,d.) (which is in the scale of the TMR channel):

$$
S_{\mathrm{AF660, \text {scaled }}}(\text { utr.,d. })=S_{\mathrm{AF} 660}(\text { utr.,d. }) \times C_{\mathrm{A}}
$$

$C_{A}$ can be calculated using signals from single-labeled and double-labeled, untreated cells according to

$$
C_{\mathrm{A}}=\frac{S_{\mathrm{TMR}}(\text { utr.,s. })-S_{\mathrm{TMR}}(\text { utr.,d. })}{S_{\mathrm{AF} 660}(\text { utr., d. })}=\frac{100 \%-S_{\mathrm{TMR}}(\text { utr.,d. })}{S_{\mathrm{AF} 660}(\text { utr., d. })}
$$

where we considered that the TMR signal of single-labeled, untreated cells was scaled to $100 \%$ using eq. $\mathrm{S} 1$ before. This allows to correct the signals recorded in the AF660 channel for all treated samples according to:

$$
S_{\mathrm{AF660, \text {scaled }}}(\text { tre.,d. })=C_{\mathrm{A}} \times S_{\mathrm{AF660}}(\text { tre.,d. })
$$

Consequently, for a double-labeled, treated sample, $S_{T M R}\left(\right.$ tre.,d.) and $S_{\text {AF660,scaled }}($ tre.,d.) truly represent the abundance of internal and surface protein, respectively, and the sum 
$S_{\mathrm{AF} 660, \text { scaled }}($ tre., d. $)+S_{\mathrm{TMR}}($ tre., d. $)$ represents the amount of total protein, always relative to an untreated control sample.

\section{Data processing}

Mean fluorescence values gated of live, singlet cells were obtained using FlowJo 10.4 (FlowJo), and scaled using MATLAB R2017b (MathWorks) or Excel 2016 (Microsoft) as described above. Data were plotted with Prism 6.07 (GraphPad).

\section{Microscopy}

$100 \mu \mathrm{l}$ of cell suspension were transferred into glass-bottom $\mu$-slides (Ibidi, cat. no. 80827), $100 \mu$ DPBS was added and the slide gently rocked. On a SP5 confocal laser scanning microscope (Leica), at least three images per condition were recorded.

\section{Comparison with conventional protocols for surface protein quantification}

To compare SPIDA with conventional methods of measuring surface protein levels, HEKTREx_HT-EGFR cells were treated with EGF, and HEK-TREx_HT-HER2 cells with GA for the indicated times. Afterwards, SPIDA was performed as described above; however, for this comparison an inactivation step was included to prevent further receptor internalization, through which surface receptor levels would appear too high, due to the slower binding of the fluorescently labeled affinity reagents used for the conventional protocol. Cells were thus first washed once with $250 \mu \mathrm{l}$ PBSA50D (Dulbecco's PBS supplemented with $50 \mathrm{mM}$ sodium azide (SigmaAldrich, cat. no. 71290) and $10 \mathrm{mM}$ 2-deoxy-D-glucose (Sigma-Aldrich, cat. no. D8375)), and then incubated for $1 \mathrm{~h}$ in $250 \mu$ fresh PBSA50D at $37^{\circ} \mathrm{C}$ for inactivation. For detection by the conventional method, the two sequential labeling steps in SPIDA were replaced by a single incubation for 30 min with fluorescently labeled affinity reagents. Surface-localized EGFR was detected using a fluorescently labeled antibody (anti-EGFR (clone ICR10) FITC conjugate, Abcam, cat. no. ab11400) diluted 1:100 (v/v); surface-localized HER2 was detected using a single-cysteine variant of affibody ZHER2 (ref. 2), which had been coupled in-house to DY647$\mathrm{P} 1$ maleimide (Dyomics, cat. no. 649P1-03) according to a previously published protocol ${ }^{3}$.

\section{Western blots}

Cells were seeded at a density of $\sim 18000 \mathrm{~cm}^{-2}$ in DMEM supplemented with $10 \%$ "tetracycline free" fetal calf serum (Bioconcept, cat. no. 2-01F28-I), 1\% (v/v) penicillin-streptomycin solution, and $150 \mathrm{\mu g} \mathrm{ml}^{-1}$ hygromycin B. After $24 \mathrm{~h}$, gene expression was induced by addition of doxycycline $\left(1 \mu \mathrm{g} \mathrm{ml}^{-1}\right)$. The next day, the supernatant was discarded and cells were washed once with Dulbecco's PBS (DPBS). Subsequently, cells were scraped in DPBS and thereafter lysed in DPBS supplemented with $1 \%(\mathrm{v} / \mathrm{v})$ Triton $\mathrm{X}-100$. Cleared lysates were analyzed for protein content by a BCA assay. Samples were equalized in protein content with DPBS and separated by reducing $4-20 \%$ SDS-PAGE, and subsequently blotted onto PVDF-FL membranes (Milli- 
pore, cat. no. IPFL00010) using a wet blotting protocol. Casein-blocked membranes were incubated overnight at $4^{\circ} \mathrm{C}$ with indicated antibodies (EGFR and phospho-EGFR, both Cell Signaling Technology, cat. no. 4267 and 4404; HER2, Merck, cat. no. OP15; EpCAM, Cell Signaling Technology, cat. no. 2626; GAPDH, Santa Cruz, cat. no. sc-32233) diluted in PBS-TC (DPBS supplemented with casein and $0.05 \%$ (v/v) Tween-20). After washing with PBS-TC, the membranes were incubated with secondary antibodies labeled for near-infrared detection (anti-mouse, Rockland Immunochemicals, cat. no. 610-732-124; anti-rabbit, Invitrogen, cat. no. A21076) for a further two hours. Finally, after washing membranes three more times with PBS-TC, fluorescence intensities were detected at 700 and $800 \mathrm{~nm}$ on an Odyssey imaging system (LI-COR Biosciences).

\section{Proliferation assay}

HEK-TREx_HT-HER2 cells were seeded in 96-well plates at a density of 5000 cells per well, and induced on the following day with doxycycline added to a final concentration of $1 \mu \mathrm{g} \mathrm{ml}^{-1}$. One day later, cells were treated with $100 \mathrm{nM}$ of trastuzumab (TZB), pertuzumab (PZB) and DARPin 6-L4-G as well as sterile DPBS as carrier control. Four days later, proliferation was measured with a Cell Proliferation XTT Kit (NeoFroxx, cat. no. 1167TT000) according to the manufacturer's instructions, and treated samples were normalized to untreated control wells.

\section{Agents for treatment}

DARPins and derived multivalent formats were expressed and purified as previously described $^{4-6}$. Geldanamycin (GA; Sigma, cat. no. SML1278), phorbol 12-myristate 13-acetate (PMA; Sigma, cat. no. P8139), mAb 225 (Ab-3; Calbiochem, cat. no. GR13L), H11 (Thermo Fisher, cat. no. MA5-13070), and MG-132 (Merck Millipore, cat. no. 474787) were diluted in DPBS, and EGF (Sigma, cat. no. E9644) was diluted in DPBS supplemented with $0.1 \%(\mathrm{w} / \mathrm{v})$ bovine serum albumin freshly for each treatment time point, in tubes with reduced protein binding (Eppendorf, cat. no. 0030108094). TZB (Trastuzumab/Herceptin) was obtained from Kantonsapotheke Zürich. PZB (Pertuzumab/Perjeta) was a kind gift from Prof. Uwe ZangemeisterWittke. The TZB-39s ${ }^{7}$ antibody fusion was expressed from a multi-promoter plasmid derived from a pYMex10 vector. Antibodies were produced and purified as reported ${ }^{8}$. 


\section{SI Figures}
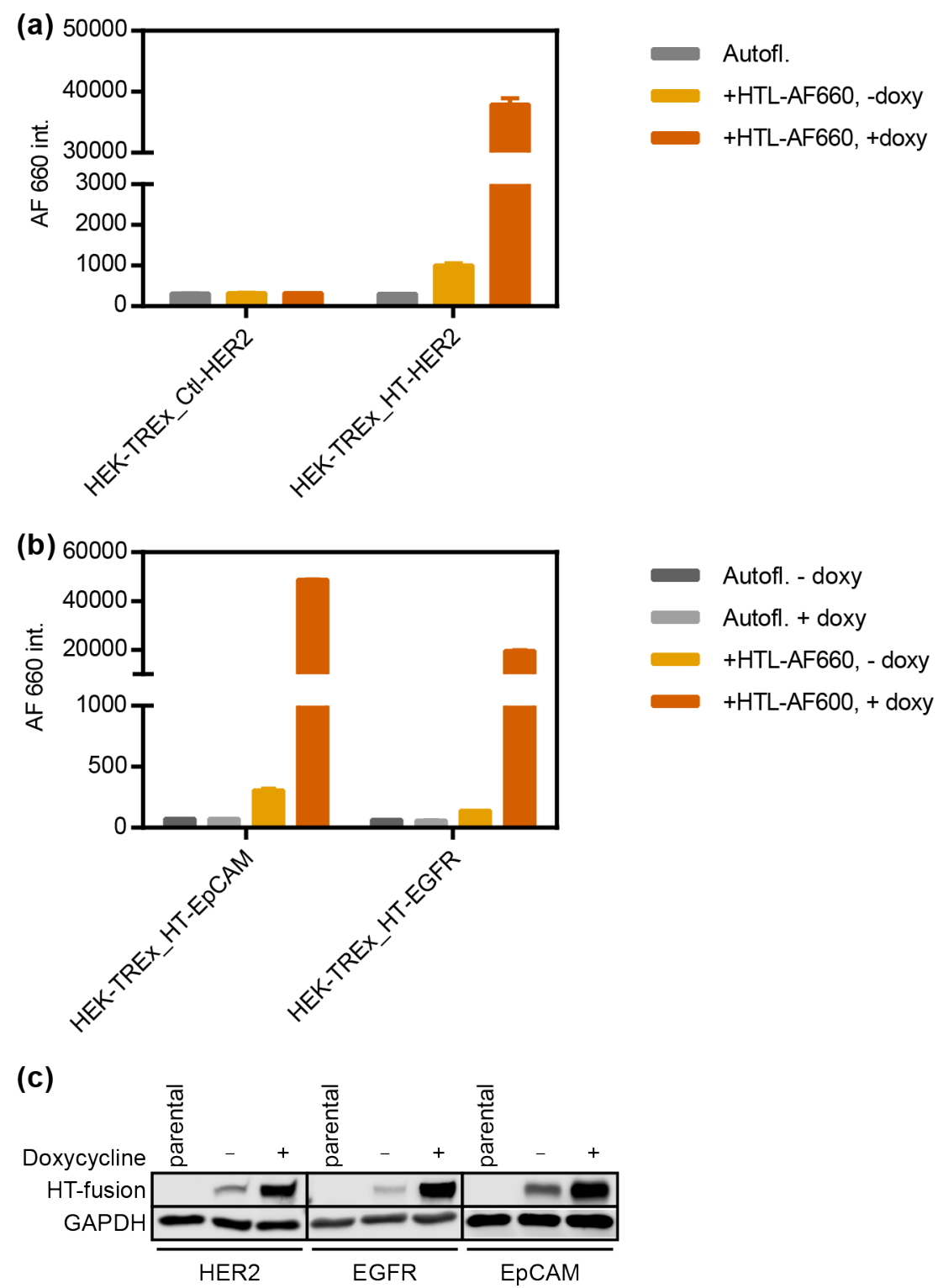

Figure S-1. Inducible expression and specific labeling of HaloTag fusions with the protein of interest (HT-POI fusions). (a) HT-HER2 expression is strongly induced by doxycycline. Nonspecific labeling of an irrelevant control tag by HTL-AF660 or background proteins is virtually absent under the chosen conditions. (b) Similar results are obtained for HT-EpCAM and HT-EGFR. (c) Western blots of cell extracts of single clones with antibodies specific for the fusion partner confirm induced expression of HaloTagged proteins. 


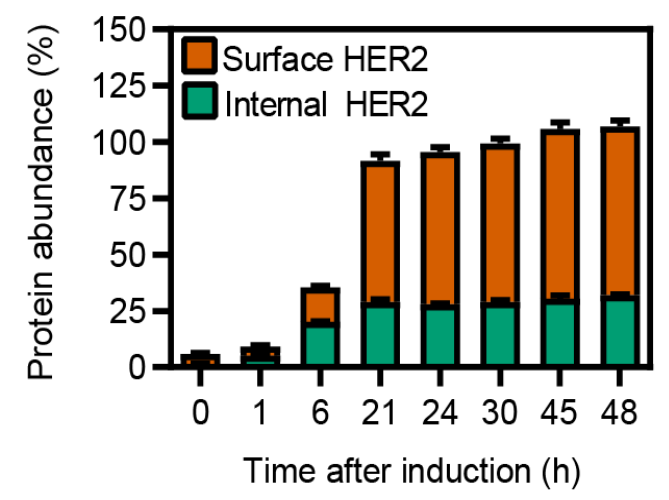

Figure S-2. Surface protein expression reaches a steady state $21 \mathrm{~h}$ after induction, which is maintained for at least until $48 \mathrm{~h}$ after induction. Fusion protein expression was induced in HEK-TREx_HT-HER2 cells by addition of doxycycline $\left(1 \mu \mathrm{g} \mathrm{ml}^{-1}\right)$ at various time points and surface, internal and total protein levels was quantified using SPIDA. 

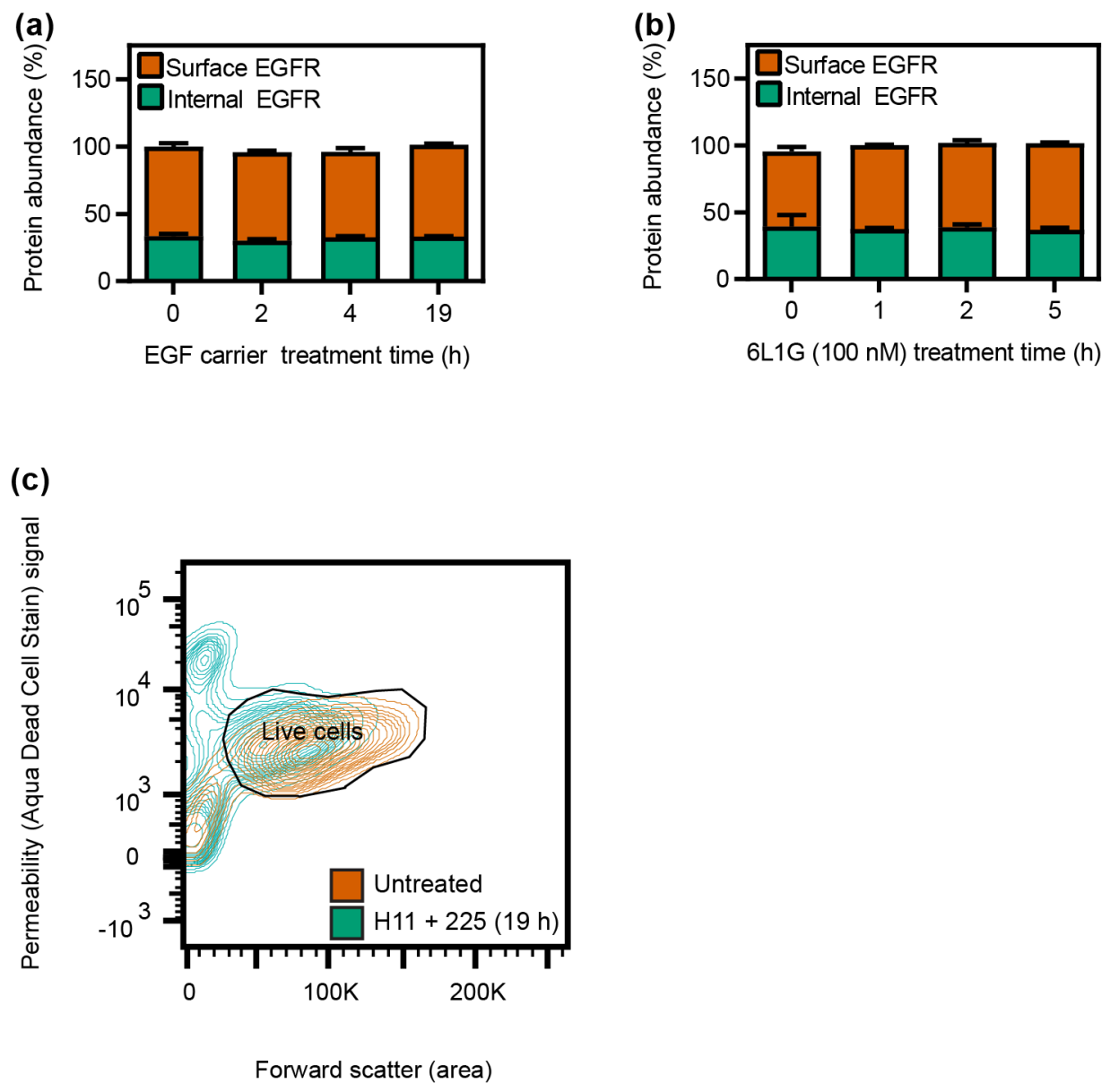

Figure S-3. Additional control treatments for HEK-TREx_HT-EGFR cells. The EGF carrier solution (containing $0.1 \%(\mathrm{w} / \mathrm{v})$ bovine serum albumin) is inert (a). HT-EGFR overexpressing cells are not affected by the biparatopic, HER2-binding DARPin 6L1G (b). Combination treatment with mAbs H11 and 225 (cf. Figure 3 ) significantly increases the fraction of dead (permeable) cells (c). 



Figure S-4. Internalization and degradation measurements for various control treatments on HEKTREx_HT-HER2 cells. Proteasome inhibitor MG-132 alone (a), antibodies PZB (b) and TZB (c), EGF (d), and EGF together with DARPin 6L1G (e) all do not significantly affect the HER2 distribution. 


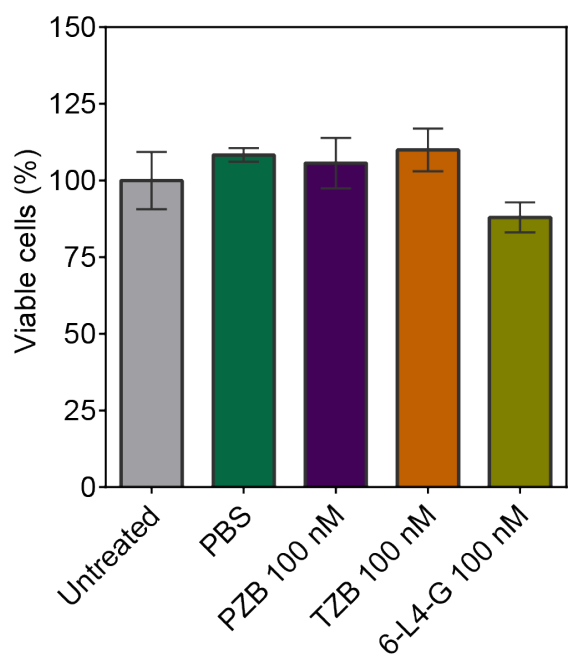

Figure S-5. Four-day proliferation assay in induced HEK-TREx_HT-HER2 cells. The clinically relevant antibodies TZB and PZB show no effect, and viability of DARPin-treated cells is reduced only slightly, as HEK cells are not HER2-addicted. 


\section{SI References}

(1) Verdurmen, W. P. R., Luginbühl, M., Honegger, A., and Plückthun, A. (2015) Efficient cellspecific uptake of binding proteins into the cytoplasm through engineered modular transport systems. J. Controlled Release 200, 13-22.

(2) Orlova, A., Magnusson, M., Eriksson, T. L., Nilsson, M., Larsson, B., Hoiden-Guthenberg, I., Widstrom, C., Carlsson, J., Tolmachev, V., Stahl, S., and Nilsson, F. Y. (2006) Tumor imaging using a picomolar affinity her2 binding affibody molecule. Cancer Res. 66, 4339-48.

(3) Hansen, S., Stüber, J. C., Ernst, P., Koch, A., Bojar, D., Batyuk, A., and Plückthun, A. (2017) Design and applications of a clamp for green fluorescent protein with picomolar affinity. Sci. Rep. 7 , 16292.

(4) Jost, C., Schilling, J., Tamaskovic, R., Schwill, M., Honegger, A., and Plückthun, A. (2013) Structural basis for eliciting a cytotoxic effect in her2-overexpressing cancer cells via binding to the extracellular domain of her2. Structure 21, 1979-1991.

(5) Zahnd, C., Kawe, M., Stumpp, M. T., de Pasquale, C., Tamaskovic, R., Nagy-Davidescu, G., Dreier, B., Schibli, R., Binz, H. K., Waibel, R., and Plückthun, A. (2010) Efficient tumor targeting with high-affinity designed ankyrin repeat proteins: Effects of affinity and molecular size. Cancer Res. 70, 1595-1605.

(6) Stefan, N., Martin-Killias, P., Wyss-Stoeckle, S., Honegger, A., Zangemeister-Wittke, U., and Plückthun, A. (2011) Darpins recognizing the tumor-associated antigen epcam selected by phage and ribosome display and engineered for multivalency. J. Mol. Biol. 413, 826-843.

(7) Li, J., Dimasi, N., Coats, S. R., Damschroder, M., Gao, C., Rainey, G., Gao, C., Toader, D., Gingipalli, L., Wang, F., Fleming, R., Bezabeh, B., Yuan, A. Q., and Kasturirangan, S. Bispecific her2 antibodies. US15302018, 2015.

(8) Nemergut, M., Žoldák, G., Schaefer, J. V., Kast, F., Miškovský, P., Plückthun, A., and Sedlák, E. (2017) Analysis of igg kinetic stability by differential scanning calorimetry, probe fluorescence and light scattering. Protein Sci. 26, 2229-2239.

(9) Spangler, J. B., Neil, J. R., Abramovitch, S., Yarden, Y., White, F. M., Lauffenburger, D. A., and Wittrup, K. D. (2010) Combination antibody treatment down-regulates epidermal growth factor receptor by inhibiting endosomal recycling. Proc. Natl. Acad. Sci. U. S. A. 107, 13252-13257. 